\title{
A Novel CCM2 Missense Variant Caused Cerebral Cavernous Malformations in a Chinese Family
}

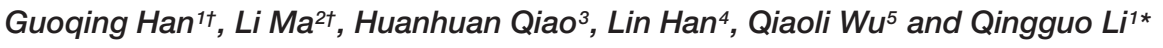 \\ ${ }^{1}$ Department of Neurosurgery, Tianjin Huanhu Hospital, Tianjin, China, ${ }^{2}$ Department of Preventive Dentistry, School \\ of Stomatology, Tianjin Medical University, Tianjin, China, ${ }^{3}$ Tianjin Key Laboratory of Brain Science and Neural Engineering, \\ Academy of Medical Engineering and Translational Medicine, Tianjin University, Tianjin, China, ${ }^{4}$ Running Gene Inc., Beijing, \\ China, ${ }^{5}$ Tianjin Neurosurgical Institute, Tianjin Huanhu Hospital, Tianjin, China
}

OPEN ACCESS

Edited by:

Fabio Coppedè,

University of Pisa, Italy

Reviewed by:

Jun Zhang,

Texas Tech University Health Sciences Center, United States

Robert Shenkar,

University of Chicago, United States

*Correspondence:

Qingguo $L$

lag369@126.com

${ }^{t}$ These authors have contributed equally to this work

Specialty section:

This article was submitted to Neurogenomics, a section of the journal

Frontiers in Neuroscience

Received: 09 September 2020

Accepted: 12 November 2020

Published: 05 January 2021

Citation:

Han G, Ma L, Qiao H, Han L, Wu Q and Li Q (2021) A Novel CCM2

Missense Variant Caused Cerebral

Cavernous Malformations in a

Chinese Family.

Front. Neurosci. 14:604350.

doi: 10.3389/fnins.2020.604350
Cerebral cavernous malformations (CCMs) are common vascular malformations in the central nervous system. Familial CCMs (FCCMs) are autosomal dominant inherited disease with incomplete penetrance and variable symptoms. Mutations in the KRIT1, CCM2, and PDCD10 genes cause the development of FCCM. Approximately 476 mutations of three CCM-related genes have been reported, most of which were case reports, and lack of data in stable inheritance. In addition, only a small number of causative missense mutations had been identified in patients. Here, we reported that $8 / 20$ members of a Chinese family were diagnosed with CCMs. By direct DNA sequencing, we found a novel variant $c .331 \mathrm{G}>\mathrm{C}(\mathrm{p} . \mathrm{A} 111 \mathrm{P})$ in exon 4 of the CCM2 gene, which was a heterozygous exonic variant, in 7/20 family members. We consider this variant to be causative of disease due to a weaken the protein-protein interaction between KRIT1 and CCM2. In addition, we also found the exon 13 deletion in KRIT1 coexisting with the CCM2 mutation in patient IV-2, and this was inherited from her father (patient III-1H). This study of a Chinese family with a large number of patients with CCMs and stable inheritance of a CCM2 mutation contributes to better understanding the spectrum of gene mutations in CCMs.

\footnotetext{
Keywords: familial cerebral cavernous malformation, CCM2, missense variant, chinese family, susceptibilityweighted imaging
}

\section{INTRODUCTION}

Cerebral cavernous malformations (CCMs) are the second most prevalent type of vascular malformation characterized by clustered enlarged capillary-like vessels and the absence of intervening neural tissue in the central nervous system (Rigamonti et al., 1988). The diameters of developmental venous anomalies range from a few millimeters to several centimeters (Rigamonti et al., 1988; Gil-Nagel et al., 1995). With an incidence of $0.1-0.5 \%$, CCMs represent 10 to $20 \%$ of all cerebrovascular malformations (Flemming et al., 2017; Scimone et al., 2017). Clinical manifestations most often occur between 20 and 30 years old. These patients most commonly experience seizures (40-70\%), focal neurologic deficits without intracranial bleeding (25-50\%), non-specific headaches (10-30\%), and intracranial hemorrhage (25-32\%) (Akers et al., 2017). However, approximately $40 \%$ of all CCM patients are asymptomatic (Labauge et al., 2007). The majority of CCMs are sporadic and comprise a single lesion, while the rest (approximately 20\%) 
are familial CCMs (FCCMs), which are caused by autosomal dominant inherited mutations and are usually associated with multiple lesions (Zafar et al., 2019).

In FCCMs, three related genes, namely CCM1 (Krev interaction trapped-1, KRIT1), CCM2 (MGC4607), and CCM3 (programmed cell death protein 10, PDCD10), have been reported (Dupre et al., 2003; Bergametti et al., 2005). According to the Human Gene Mutation Database (HGMD) (updated through December 2019), there are 476 germline mutations of the three genes that have been described as causative mutations of CCMs (Scimone et al., 2018). A large majority of causative mutations include non-sense, frameshift, and splicing mutations, commonly resulting in a premature stop codon and dysfunctional mRNA (Zafar et al., 2019). However, only a small number of causative missense mutations have been identified in patients, and most of them lead to abnormalities in splicing (Riant et al., 2013; Bergametti et al., 2020).

Herein, we reported a large Chinese family with multiple members having CCMs; two patients were seen in our institute, and we summarized the clinical and radiological features of 20 members from this family. We screened CCM-related genes through whole-exome sequencing (WES) and identified a novel missense variant (c.331G > C) in CCM2 that caused at least seven cases of CCMs; we also identified the exon 13 deletion in KRIT1 coexisting with the CCM2 mutation in patient IV-2 and her father (patient III-1H). This study contributes to better understanding the spectrum of mutations of FCCM.

\section{MATERIALS AND METHODS}

\section{Whole-Exome Sequencing}

Peripheral blood samples were collected from members of the family and then sent to Running Gene Inc (Beijing, China) for sequencing. WES was performed and included the targeted KRIT1, CCM2, and PDCD10 genes. DNA samples were extracted by a DNA Isolation Kit (AU1802, BioTeke, China), concentrated using a Qubit dsDNA HS Assay Kit (Q32851, Invitrogen, United States), purified, and PCR amplified with Agencourt AMPure XP beads. Targeted DNA samples were sequenced on HiSeq X10 (Illumina, San Diego, United States). All variants were filtered first against the 1000 Genomes Project database for a minor allele frequency (MAF) $\geq 1 \%$ and $\mathrm{ExAC}$ hom $\mathrm{AC} \geq 3$. The obtained variants were further selected according to co-segregation, genetic model, and MAF $<1 \%$ in three databases $(1000$ Genomes Project_EAS, ExAC, gnomAD_EAS).

According to the American College Medical Genetics and Genomics (ACMG) guidelines, mutations was analyzed to assess the risk of pathogenicity (Richards et al., 2015). Sanger sequencing was used to validate the family segregation of mutations. The variants were identified as novel when they were not reported in previous literatures or were not found in the following public databases: (1) CCM mutation database $^{1}$ (2) dbSNP at the National Center for Biotechnology

${ }^{1}$ https://www.angioma.org/
Information $(\mathrm{NCBI})^{2}$; (3) ClinVar at $\mathrm{NCBI}^{3}$, and (4) $\mathrm{HGMD}^{4}$ (Huang et al., 2016).

\section{RESULTS}

\section{Case Presentation}

The proband (II-1) was a 60-year-old woman who had complained of an inability to walk for 10 years, and was admitted to our hospital because of hypophrasia, and loss of consciousness for 2 days. The left lateral ventricle and subcortical regions of the cerebral hemispheres were showed multiple high-intensity patchy calcifications or bleeding from computed tomography (CT) findings (Figures 1A,B). Gradient-recalled echo (GRE) T2weighted magnetic resonance imaging (MRI) showed a large number of dark spots, and mass of different sizes distributed in the left lateral ventricle, bilateral cerebral hemisphere, and cerebellum (Figures 1C,D), which indicated multiple cavernous malformations. The patient received an operation on the left lateral ventricle lesion. However, her symptoms were not improved shortly. Conversely, she suffered the complications of hypothalamic diabetes insipidus, pulmonary infection, and loss of consciousness for a long time. Pathological observations showed vascular malformations (mixed type) associated with bleeding, calcification, and iron deposits (Figures 1E,F). The Ki67 proliferation rate was $2.6 \%$. After nearly a month of treatment, she gradually regained consciousness.

Considering that multiple CCMs usually occur in FCCMs, we present the family history and brain MRI examination results. Surprisingly, we found high morbidity $(40 \%, 8 / 20)$ in four generations. Here, we display the pedigree of the Chinese family in Figure 2, and all of the detailed clinical information is listed in Table 1.

Patient I-2, aged 83 years, was diagnosed with CCM approximately 6 years ago. Susceptibility-weighted imaging (SWI) of the brain showed hundreds of dark spots and mass of different sizes distributed in the cerebral hemisphere and cerebellum (Figures 3A-C) combined with meningioma at the right parietal lobe. The diameters of the CCM lesions ranged from $1 \mathrm{~mm}$ to $3 \mathrm{~cm}$. She didn't receive any treatment and unfortunately died 1 month before we conducted this study. Patient II5 was a 41-year-old woman, showing dizziness and epileptic seizures for 3 days. Non-contrast CT revealed a high-intensity calcification or bleeding located in the right lateral ventricle with asymmetrical ventricles (Figure $3 \mathbf{H}$ ). T2-weighted gradient echo (GE) sequences showed that multiple CCM lesions were distributed in the right lateral ventricle and across the hemisphere without contrast (Figures 3I,J). Patient II-5 underwent resection of the right lateral ventricle lesion. The seizures in this patient subsided, but this treatment caused hemiplegia of the left limb. Patient IV-1, aged 13 years, complained of intermittent headaches and was diagnosed with CCM since she was 6 years old. T2-weighted MRI showed lesions on the left temporal

\footnotetext{
${ }^{2}$ http://www.ncbi.nlm.nih.gov/snp/

${ }^{3}$ https://www.ncbi.nlm.nih.gov/clinvar/

${ }^{4}$ http://www.hgmd.org/
} 

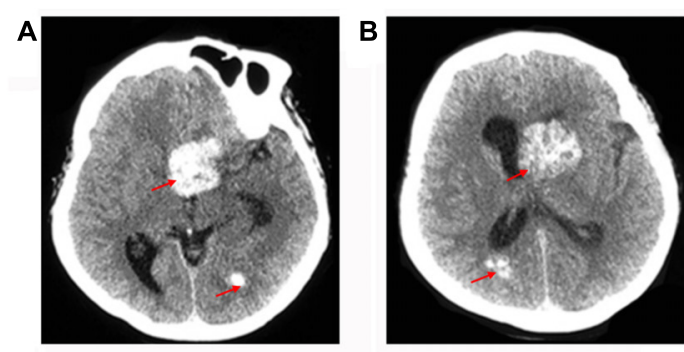

E

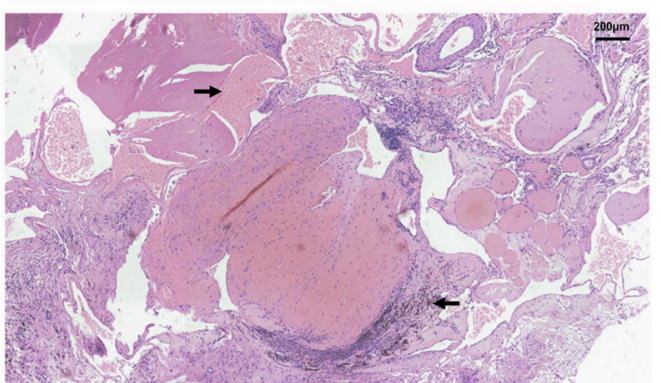

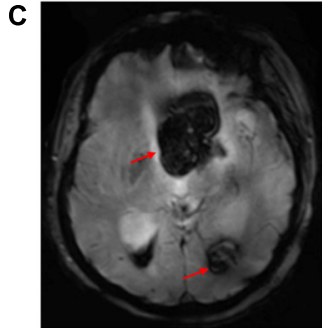
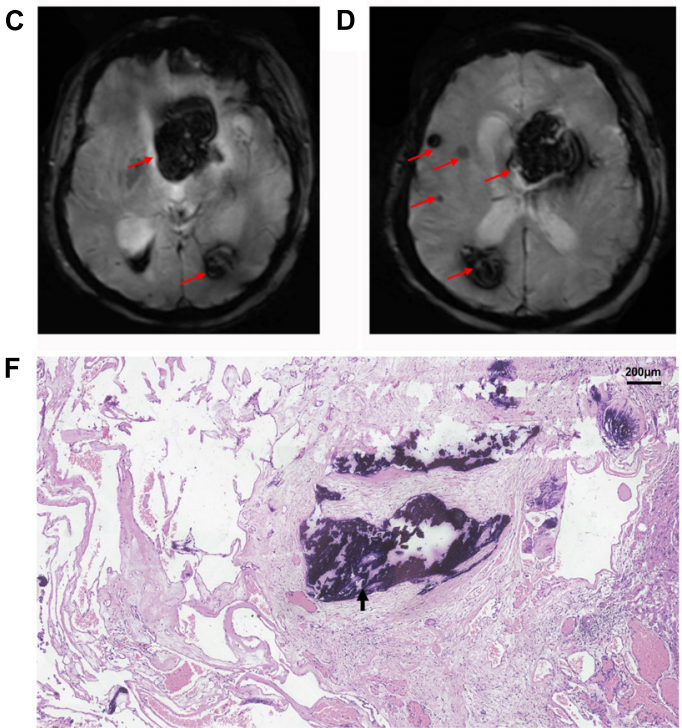

FIGURE 1 | Radiological and histopathological information of patient II-1. CT (A,B) and GRE T2-weighted MRI (C,D) showed multiple cavernous malformation lesions across the left lateral ventricle and bilateral cerebral hemisphere in the proband, patient II-1. The red arrows show the location of lesions. Histopathological examination revealed vascular malformation associated with bleeding $(\rightarrow)$, calcification $(\uparrow)$, and iron deposits $(\leftarrow)$ in the proband, patient II-1, at $(\mathbf{E}) \times 100$, and (F) $\times 100$

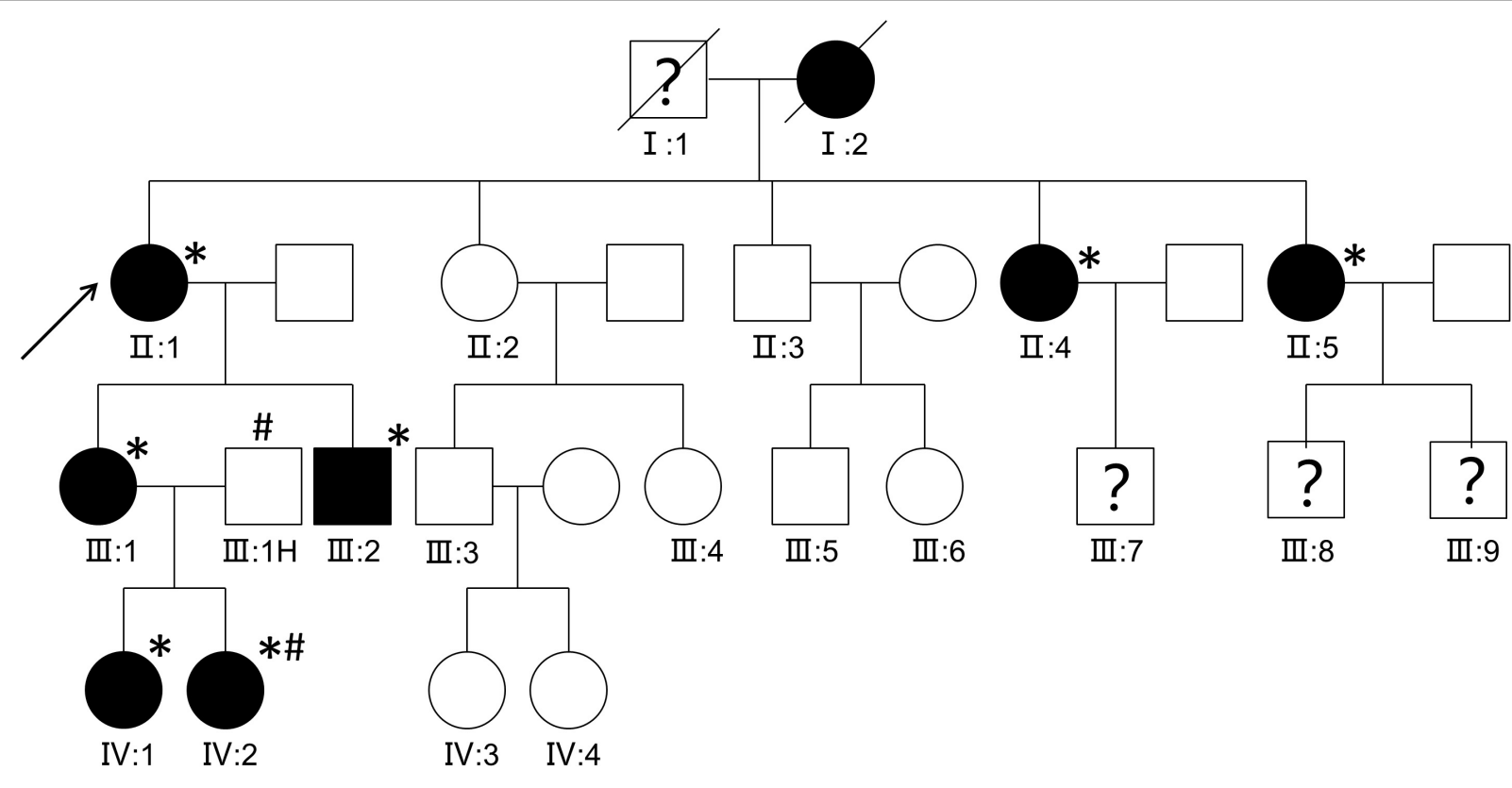

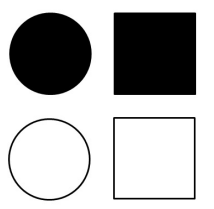

CCMs patients diagnosed by MRI

Normal in MRI

* Positive in CCM2 gene

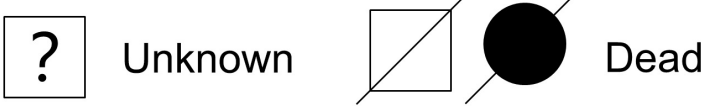

The proband

\# Positive in KRIT1 gene

FIGURE 2 | Pedigree of a Chinese family. Affected patients were diagnosed with CCMs upon T2-weighted MRI or SWI of the brain. 


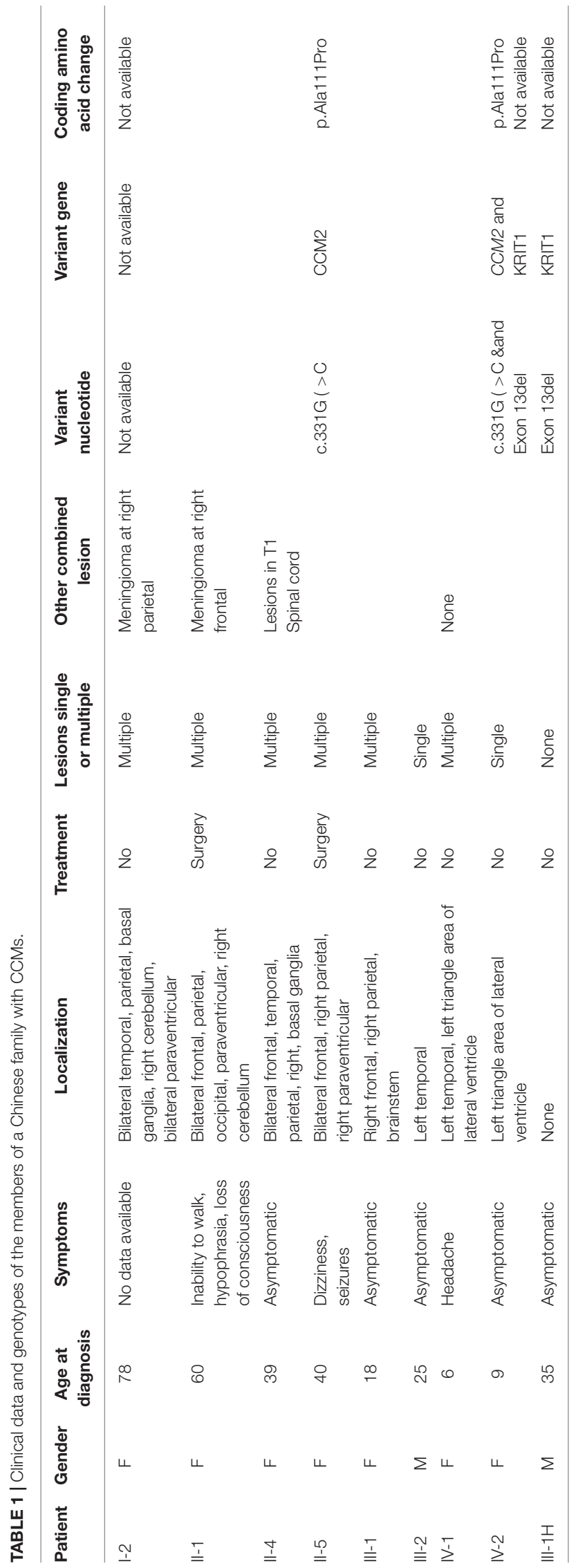

and triangular areas of the lateral ventricle (Figures 3N,O). Patient I-1 died several years ago, and the family had lost contact with patients III-7, III-8, and III-9. Therefore, their MRI data were not available. Patients II-4 (Figures 3D-G), III-1 (Figures 3K,L), III-2 (Figure 3M), and IV-2 (Figure 3P) were asymptomatic.

\section{Mutational Analysis}

WES was performed on 15 members of a Chinese family, except for patients I- 1 and I- 2 as both were dead and patients III-7, III-8, and III-9 (lost contact with the family). Detailed results are also listed in Table 1. A heterozygous variant c.331G > C (Chr7:45104104) in exon 4 of the CCM2 gene (NM_031443, NP_113631) was detected in 7/20 family members (Figures 4A,B). This variant caused the 111 st amino acid alanine to be replaced with a proline (p.A111P), and native Ala111 residue is located in the phosphotyrosine binding (PTB) domain as a putative phosphoinositide binding site (PM1). Variant c.331G $>\mathrm{C}$ is absent from public databases such as gnomAD, 1000 Genomes, ExAC (PM2), and it is also a novel mutation that has not been reported in the dbSNP, HGMD, ClinVar, and CCM mutation databases or in the previous literature. The variant co-segregated with CCMs in seven affected family members (PP1), and it is predicted as deleterious in multiple computational software (PP3), including MutationTaster2 (disease causing, probability: 1.000 ), SIFT (damaging, $0.008<0.05$ ), PROVEAN (deleterious, $-2.65<-2.5$ ), and PolyPhen-2 (probably damaging, probability: 0.999). Thus, the variant c.331G > C (p.A111P) was considered to be likely pathogenic, according to ACMG standards and guidelines (Richards et al., 2015).

The other variant, a deletion in exon 13 of KRIT1 (NM_194456), was detected in the proband's second granddaughter (IV-2), who was asymptomatic (Figure 4C). To ensure the source of this KRIT1 gene mutation, we used WES to examine the DNA from patient IV-2's father (patient III-1H) and found the identical deletion; however, patient III-1H had a normal MRI scan. The variant was a heterozygous genomic deletion mutation and has been reported in the HGMD as associated with CCM lesion development through a splicing impairment (Mondejar et al., 2014).

We used an in silico approach to test the effect of the novel c.331G > C variant of the CCM2 gene. A structural comparison of the wild-type and mutated proteins was performed by PyMOL software $^{5}$ (Figure 5). To predict the secondary structure of the polypeptide, we used an extensible molecular modeling system, the UCSF Chimera ${ }^{6}$ tool. From the in silico analysis, we found that the native Ala111 residue in the PTB domain (located in the beta 2 strand) of the malcavernin protein interacted with Val67 (located in the beta 1 strand) through two intramolecular hydrogen $(\mathrm{H})$ bonds. Once the Pro111 mutant was superimposed, a change in the number, and position of the intramolecular $\mathrm{H}$ bond of the PTB domain could cause an alteration in the folding at position 111.

\footnotetext{
${ }^{5}$ https://pymol.org/2/

${ }^{6}$ http://www.cgl.ucsf.edu/chimera/download.html
} 
A

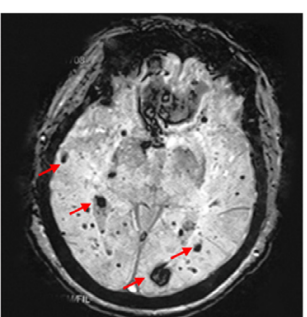

D

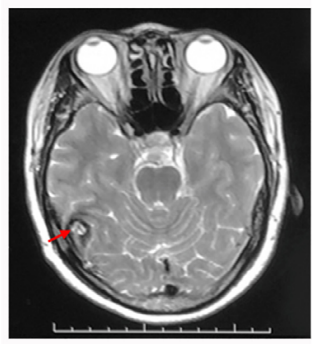

H

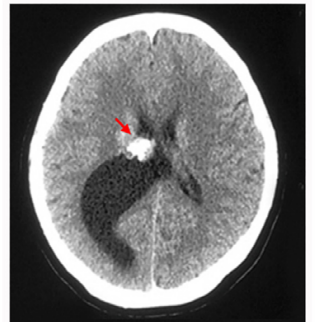

K

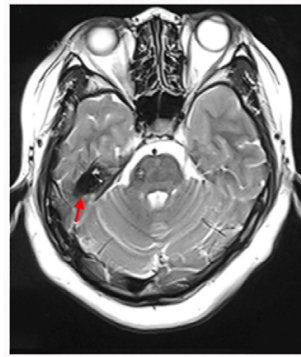

N

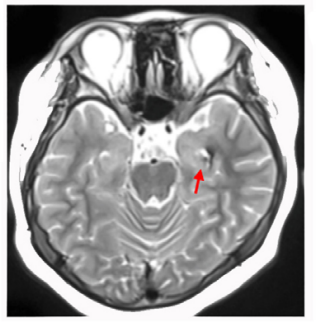

B

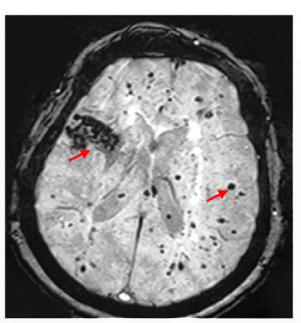

E

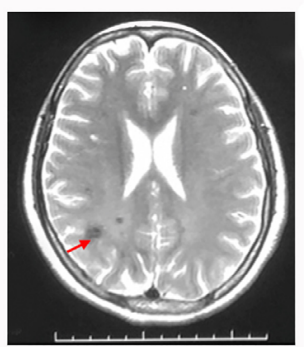

I

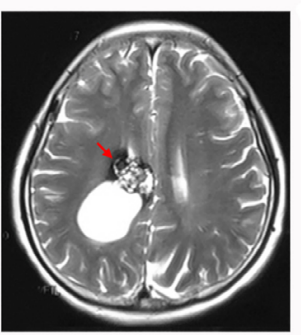

L

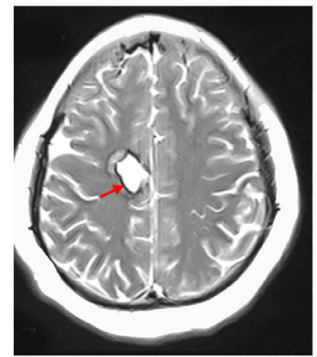

0

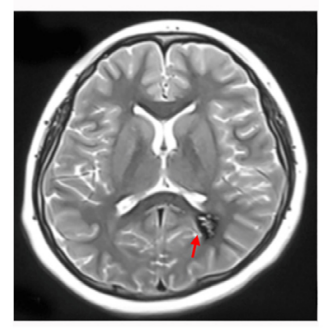

C

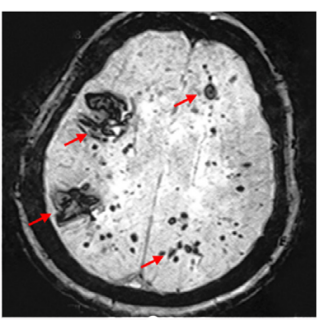

F

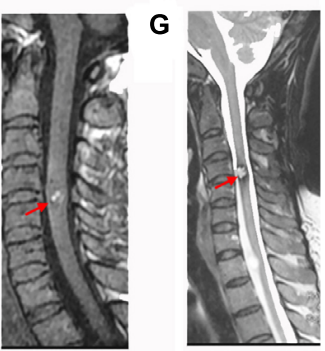

J

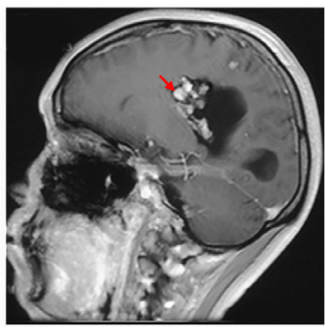

M

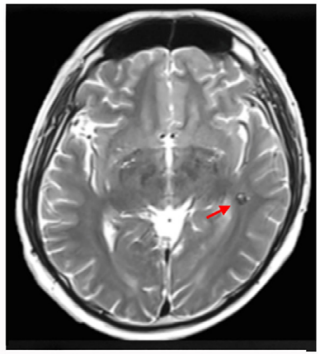

P

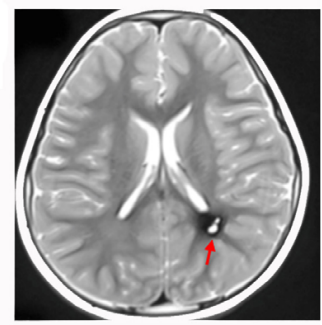

FIGURE 3 | CCM lesions diagnosed through CT, T2-weighted MRI and SWI. (A-C) SWI of patient I-2 showed hundreds of lesions distributed across the cerebral hemisphere and cerebellum. T2-weighted MRI of patient II-4 showed multiple CCMs in the right temporal lobe (D), frontal and parietal lobes (E), and spinal cord $(\mathbf{F}, \mathbf{G})$. CT (H), T2-weighted (I), and contrast MRI (J) of patient II-5 showed lesions with a "popcorn" appearance located in the right lateral ventricle and frontal-parietal lobe with asymmetrical ventricles. (K,L) T2-weighted MRI of patient III-1 showed lesions surrounded by a dark rim of hemosiderin on the right frontal and temporal lobes. (M) T2-weighted MRI of patient III-2 showed a lesion located on the left temporal lobe. (N,O) T2-weighted MRI of patient IV-1 showed lesions on the left temporal lobe and triangle area of the lateral ventricle. (P) T2-weighted MRI of patient IV-2 showed a lesion located in the left triangle area of the lateral ventricle. The red arrows show the CCM lesions.

\section{DISCUSSION}

In the 1980s, (Hayman et al., 1982) and (Rigamonti et al., 1987) suggested that FCCMs may be an autosomal dominant inherited disease with incomplete penetrance through analyzing the pedigree of an affected family. Dubovsky et al. (1995) mapped the CCM1/KRIT1 gene in 1995 as it had a high prevalence rate in Hispanic families from southwestern United States. 


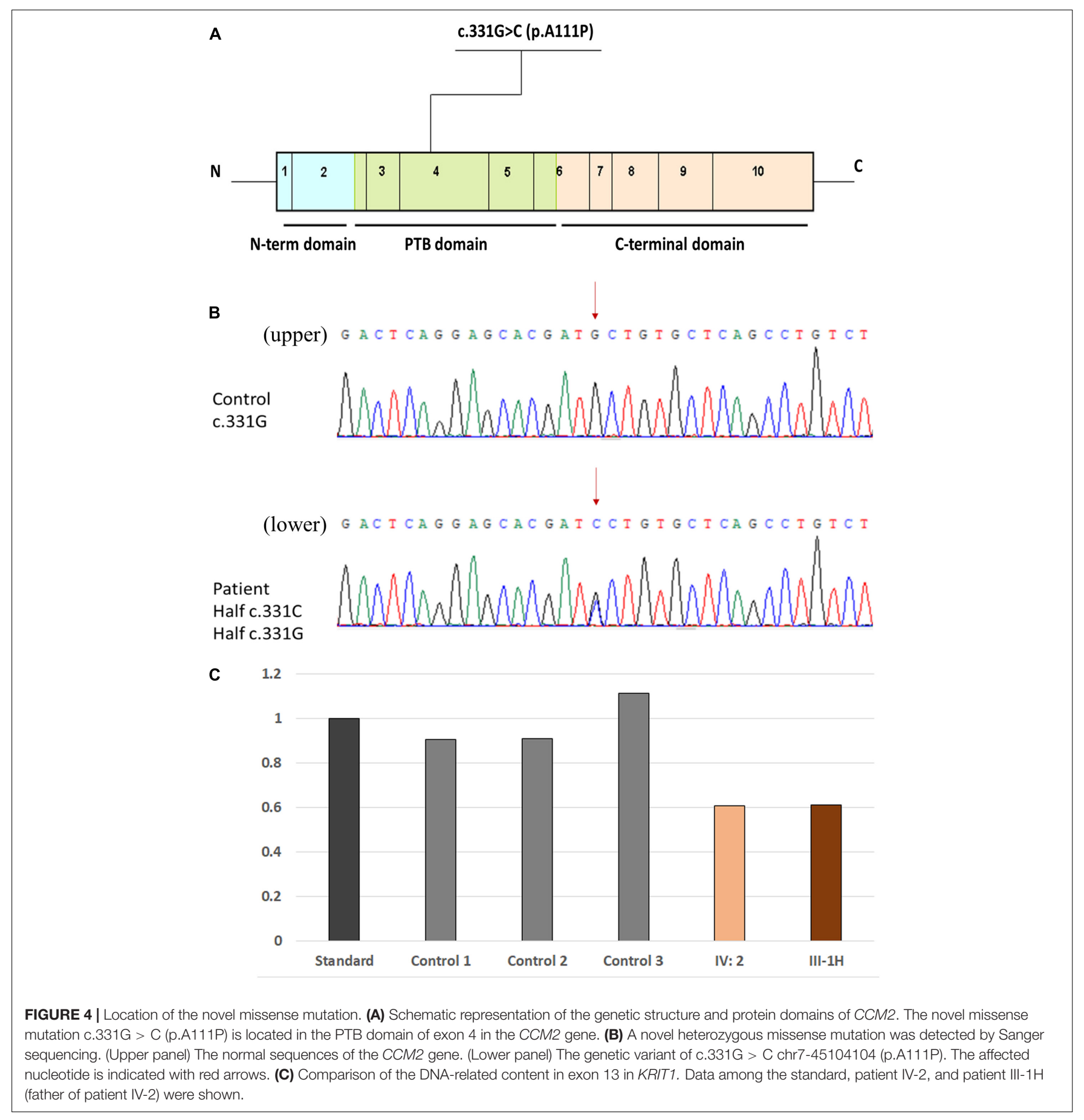

Craig et al. (1998) analyzed 20 non-Hispanic CCM families and identified and named two new CCM-related genes, CCM2, and $C C M 3 / P D C D 10$. Previous studies have shown that the prevalence of mutations ranges from $70 \sim 90 \%$ to $16 \sim 60 \%$ in familial and sporadic CCMs, respectively (Spiegler et al., 2014; Merello et al., 2016), in which the KRIT1, CCM2, and PDCD10 genes account for $60 \sim 65 \%, 18 \sim 19 \%$, and $16 \sim 22 \%$, respectively (Riant et al., 2013; Mondejar et al., 2014; Spiegler et al., 2014). KRIT1 mutations usually have a favorable clinical prognosis, while PDCD10 mutations seem to show a more severe phenotype characterized by recurrent intracranial hemorrhage at a younger age (Shenkar et al., 2015).

In our study, seven members of the family carried the missense mutation c.331G > C, and all of them showed lesions on an MRI scan. Members without this mutation did not have lesions on an MRI scan. Clinical penetrance was estimated to be nearly $88 \%$ for KRIT1, $100 \%$ for CCM2, and $63 \%$ for PDCD10 (de Vos et al., 2017). And CCM2 is the only gene which was 

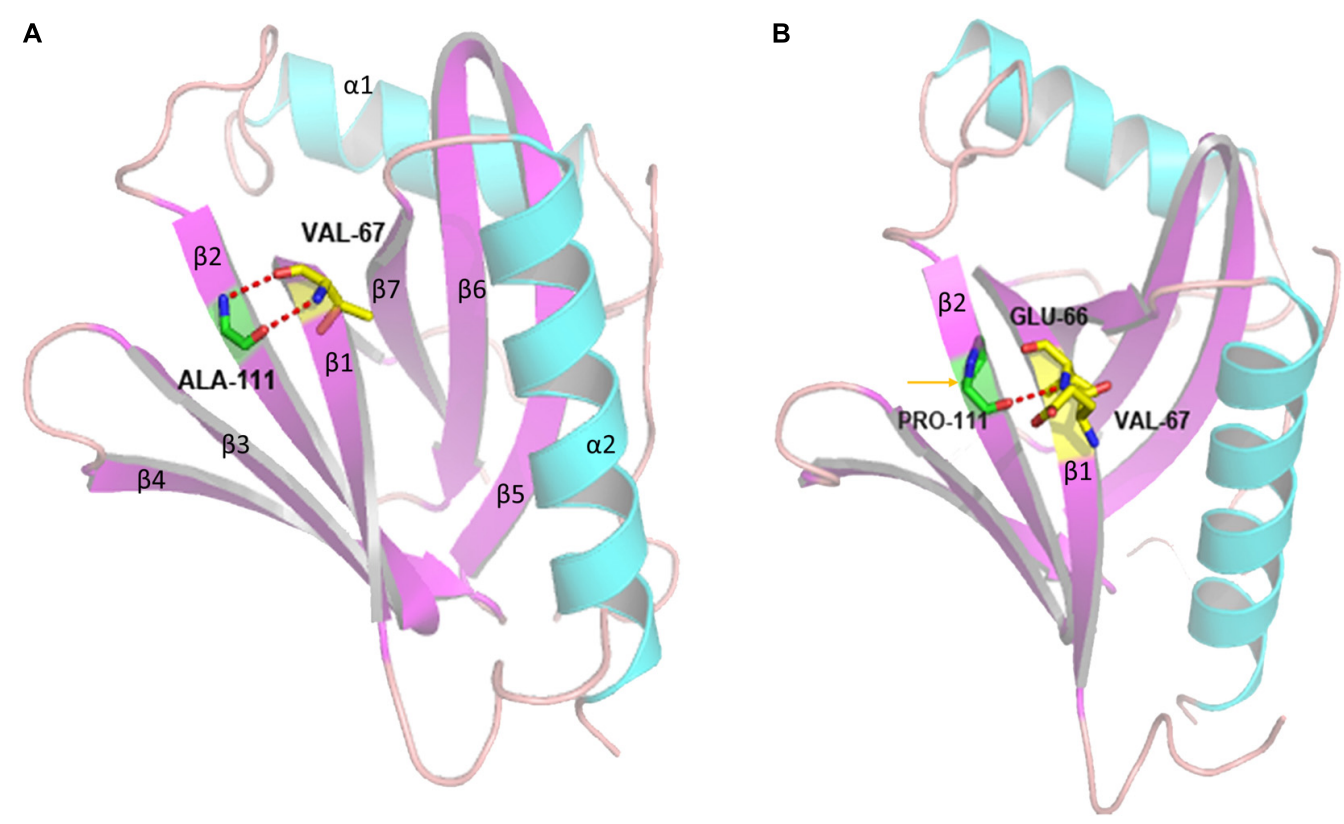

FIGURE 5 | Three-dimensional structure of the PTB domain generated by PyMOL software. The panel shows the overlap between the wild-type (A) and mutated (B) tertiary protein structures. p.A111P: superimposed structures of the malcavernin protein with the native Ala111 residue and Pro111 residue substitution. The yellow arrow indicates the region where the A111P substitution resides, and the dotted red lines indicate the alteration in the intramolecular $\mathrm{H}$ bonds in the area of the substitution.

reported to cause $100 \%$ radiological changes, which means that the offspring have a 50\% risk of developing the CCM disease if one of the parents carries the c.331G $>\mathrm{C}$ mutation. The majority of patients $(7 / 8)$ in this study were female, suggesting that CCM2 had a higher incidence in women. The ages at diagnosis ranged from 6 to 78 years old, and the median age of onset was 32 years old. Among the affected members (8/20), 3 patients complained of clinical symptoms. Patient IV-1 had a headache, while others were admitted due to focal nerve dysfunctions. Most (5/8) were asymptomatic and diagnosed only by an MRI examination. Patients with CCMs have heterogeneous phenotypes. Studies focused on genotype-phenotype correlations found a high variability of symptoms, even in patients belonging to the same family, and harboring the same mutation (Scimone et al., 2018). Heterogeneity of clinical manifestations and onset age were considered to be associated with the incomplete penetrance of the disease (Battistini et al., 2007).

Multiple lesions frequently occur in FCCMs and can be distributed in both the supratentorial and infratentorial regions. Considering a two-hit hypothesis for CCM pathogenesis, the different locations of lesions may be explained by the stochastic nature of somatic mutations (Akers et al., 2009). Among the patients with MRI data, two had a single brain lesion (III-2 and IV-4), and one had a lesion in the spinal cord (II-4), implying that even a single lesion, or a spinal lesion can be associated to the FCCMs, and the patients should be advised to a genetic counseling service (Cigoli et al., 2015).

Three patients (I-2, II-1, and II-4) also had other diseases, such as meningioma, suggesting that there may be some similarities in the development of the disease. In addition, we found a declining trend in the number of CCM lesions and complicated diseases in the four generations. The reason is still unclear, and it may be associated with the patients' self-repair functions, which need to be further explored.

The proteins KRIT1, malcavernin, and PDCD10 compose a ternary complex that is involved in maintaining cell-cell junctions and cell-extracellular matrix adhesion, regulating the proliferation, and apoptosis of endothelial cells (Zafar et al., 2019). In the structure of the complex, malcavernin contains a PTB domain that mediates the interaction with KRIT1 and PDCD10 (Zawistowski et al., 2005). CCM2 acts as a bridge between KRIT1 and PDCD10 and interacts with the juxtamembrane region of the TrkA receptor tyrosine kinase, mediating TrkA-induced death in diverse cell types (Harel et al., 2009).

The CCM2 gene encodes malcavernin, a 444 amino acid protein that contains an $\mathrm{N}$-terminal flexible loop of 60 residues, a PTB domain (encoded by residues 60 to 220), and a harmonin homology domain (HHD) at its C-terminus (residues $\sim 220$ to 444) (Fisher et al., 2013, 2015). The novel missense variant c.331G > C (p.A111P) that we identified is located in the PTB domain. Thus, we considered that the c.331G $>\mathrm{C}$ variant might cause the pathogenesis of FCCM through altering the structure and even destroying the function of the PTB domain.

From the in silico analysis, we found that c.331G $>\mathrm{C}$ (p.A111P) altered the number and position of intramolecular $\mathrm{H}-\mathrm{H}$ bonds in the PTB domain. According to the pathogenic missense variants reported in the literature (Fisher et al., 2015; Bergametti et al., 2020), all missense variants changed the original amino acid to either a proline (9/11) or an arginine (2/11). 
Proline was usually found in some tight turns in protein structures where the polypeptide chain must change direction but was rarely involved in active or binding sites of the protein. Proline is also rarely found in $\alpha$-helices and $\beta$-sheets, as it would reduce the stability of such structures, while arginine is a basic, polar amino acid that is positively charged and very hydrophilic (Bergametti et al., 2020). Furthermore, coimmunoprecipitation experiments found an absence of some amino acids located in the PTB domain KRIT1 could cause the loss of the interaction between KRIT1 and CCM2 (Fisher et al., 2015; Bergametti et al., 2020).

In addition, a deletion of exon 13 in KRIT1 was detected in patient IV-2. This variant was a heterozygous genomic deletion and had been reported in a previous study to be associated with CCM lesion development through splicing impairment (Mondejar et al., 2014). Patient IV-2 harbored two mutations in the CCM gene, both of which could cause CCMs. However, the patient has not shown any manifestations to date and presented with only a single lesion. To estimate the origins of the two mutation sites, we further sequenced the DNA from her father (patient III-1H), who had a normal MRI result. Coincidentally, we found that her father (patient III-1H) also had the deletion of exon 13 in KRIT1, indicating that patient IV-2 inherited a causative gene from each parent. Both mutations were located on chromosome 7 (KRIT1 was in the long arm, while CCM2 was in the short arm). According to the penetrance of the KRIT1 and CCM2 genes, the predicted incidence rate of the offspring of patient IV-2 is $94 \%$, but potential offspring have a $100 \%$ chance of carrying a causative gene mutation. Although no obvious symptoms were observed in this young patient, long-term followup is required to understand the ultimate pathogenicity of the relevant genes.

\section{CONCLUSION}

We reported a Chinese family with four generations carrying the c.331G > C variant in exon 4 of CCM2, and this is a novel missense variant that causes CCMs. The novel variant in the CCM2 gene showed autosomal dominant heredity and had $100 \%$ neuroradiological penetrance. This study of a family with a large number of patients with CCMs and stable inheritance of a CCM2 mutation contributes to better understanding the spectrum of gene mutations in CCMs. We consider this variant to be causative of disease, but additional immunoprecipitation experiments will be needed to evaluate its pathogenesis. Specifically, patient IV-2 carries two causative mutations, including exon 13 deletion in KRIT1 and c.331G > C

\section{REFERENCES}

Akers, A., Al-Shahi Salman, R., Awad, I. A., Dahlem, K., Flemming, K., Hart, B., et al. (2017). Synopsis of guidelines for the clinical management of cerebral cavernous malformations: consensus recommendations based on systematic literature review by the angioma alliance scientific advisory board clinical experts panel. Neurosurgery 80:5.

Akers, A. L., Johnson, E., Steinberg, G. K., Zabramski, J. M., and Marchuk, D. A. (2009). Biallelic somatic and germline mutations in cerebral cavernous in CCM2, with approximately a $100 \%$ predicted incidence rate in offspring. More advice on prenatal guidance and long-term follow-up are needed.

\section{DATA AVAILABILITY STATEMENT}

The datasets presented in this study can be found in online repositories. All the whole-exome sequencing cDNA (Illumina) data from this study have been submitted to the GenBank of NIH genetic sequence database (https://www. ncbi.nlm.nih.gov/WebSub/?form=history\&tool=genbank) under accession number PRJNA639922.

\section{ETHICS STATEMENT}

The studies involving human participants were reviewed and approved by the Research Ethics Committee of Tianjin Huanhu Hospital. Written informed consent to participate in this study was provided by the participants' legal guardian/next of kin. Written informed consent was obtained from the individual(s), and minor(s)' legal guardian/next of kin, for the publication of any potentially identifiable images or data included in this article.

\section{AUTHOR CONTRIBUTIONS}

GH and QL conceived the study. GH, LM, and HQ performed the clinical evaluation. QW performed the imageological assessment and MRI examination of patients. LH collected clinical information and also contributed to the sequencing, validation and analysis of novel mutations. $\mathrm{GH}$ and $\mathrm{LH}$ polished the figures and tables. GH drafted the manuscript. LH, HQ, and $\mathrm{QL}$ edited the manuscript. All authors read and approved the final manuscript.

\section{FUNDING}

This work was supported by the Tianjin Natural Science Foundation (18ZXDBSY00180) and Tianjin Health and Health Committee Science and Technology Project (QN20009).

\section{ACKNOWLEDGMENTS}

The authors thank all the participants in this family for their contribution to the study.

malformations (CCMs): evidence for a two-hit mechanism of CCM pathogenesis. Hum. Mol. Genet. 18, 919-930. doi: 10.1093/hmg/ddn430

Battistini, S., Rocchi, R., Cerase, A., Citterio, A., Tassi, L., Lando, G., et al. (2007). Clinical, magnetic resonance imaging, and genetic study of 5 Italian families with cerebral cavernous malformation. Arch. Neurol. 64, 843-848. doi: 10.1001/ archneur.64.6.843

Bergametti, F., Denier, C., Labauge, P., Arnoult, M., Boetto, S., Clanet, M., et al. (2005). Mutations within the programmed cell death 10 gene cause cerebral cavernous malformations. Am. J. Hum. Genet. 76, 42-51. doi: 10.1086/426952 
Bergametti, F., Viot, G., Verny, C., Brechard, M. P., Denier, C., Labauge, P., et al. (2020). Novel CCM2 missense variants abrogating the CCM1-CCM2 interaction cause cerebral cavernous malformations. J. Med. Genet. 57, 400-404. doi: 10.1136/jmedgenet-2019-106401

Cigoli, M. S., De Benedetti, S., Marocchi, A., Bacigaluppi, S., Primignani, P., Gesu, G., et al. (2015). A novel MGC4607/CCM2 gene mutation associated with cerebral spinal and cutaneous cavernous angiomas. J. Mol. Neurosci. 56, 602-607. doi: 10.1007/s12031-015-0555-0

Craig, H. D., Gunel, M., Cepeda, O., Johnson, E. W., Ptacek, L., Steinberg, G. K., et al. (1998). Multilocus linkage identifies two new loci for a mendelian form of stroke, cerebral cavernous malformation, at 7p15-13 and 3q25.2-27. Hum. Mol. Genet. 7, 1851-1858. doi: 10.1093/hmg/7.12.1851

de Vos, I. J., Vreeburg, M., Koek, G. H., and van Steensel, M. A. (2017). Review of familial cerebral cavernous malformations and report of seven additional families. Am. J. Med. Genet. A 173, 338-351. doi: 10.1002/ajmg.a. 38028

Dubovsky, J., Zabramski, J. M., Kurth, J., Spetzler, R. F., Rich, S. S., Orr, H. T., et al. (1995). A gene responsible for cavernous malformations of the brain maps to chromosome 7q. Hum. Mol. Genet. 4, 453-458. doi: 10.1093/hmg/4. 3.453

Dupre, N., Verlaan, D. J., Hand, C. K., Laurent, S. B., Turecki, G., Davenport, W. J., et al. (2003). Linkage to the CCM2 locus and genetic heterogeneity in familial cerebral cavernous malformation. Can. J. Neurol. Sci. 30, 122-128. doi: 10.1017/s0317167100053385

Fisher, O. S., Liu, W., Zhang, R., Stiegler, A. L., Ghedia, S., Weber, J. L., et al. (2015). Structural basis for the disruption of the cerebral cavernous malformations 2 (CCM2) interaction with Krev interaction trapped 1 (KRIT1) by diseaseassociated mutations. J. Biol. Chem. 290, 2842-2853. doi: 10.1074/jbc.m114. 616433

Fisher, O. S., Zhang, R., Li, X., Murphy, J. W., Demeler, B., and Boggon, T. J. (2013). Structural studies of cerebral cavernous malformations 2 (CCM2) reveal a folded helical domain at its C-terminus. FEBS Lett. 587, 272-277. doi: 10. 1016/j.febslet.2012.12.011

Flemming, K. D., Graff-Radford, J., Aakre, J., Kantarci, K., Lanzino, G., Brown, R. D. Jr., et al. (2017). Population-based prevalence of cerebral cavernous malformations in older adults: mayo clinic study of aging. JAMA Neurol. 74, 801-805. doi: 10.1001/jamaneurol.2017.0439

Gil-Nagel, A., Wilcox, K. J., Stewart, J. M., Anderson, V. E., Leppik, I. E., and Rich, S. S. (1995). Familial cerebral cavernous angioma: clinical analysis of a family and phenotypic classification. Epilepsy Res. 21, 27-36. doi: 10.1016/ 0920-1211(95)00005-u

Harel, L., Costa, B., Tcherpakov, M., Zapatka, M., Oberthuer, A., Hansford, L. M., et al. (2009). CCM2 mediates death signaling by the TrkA receptor tyrosine kinase. Neuron 63, 585-591. doi: 10.1016/j.neuron.2009. 08.020

Hayman, L. A., Evans, R. A., Ferrell, R. E., Fahr, L. M., Ostrow, P., and Riccardi, V. M. (1982). Familial cavernous angiomas: natural history and genetic study over a 5-year period. Am. J. Med. Genet. 11, 147-160. doi: 10.1002/ajmg. 1320110205

Huang, W. Q., Lu, C. X., Zhang, Y., Yi, K. H., Cai, L. L., Li, M. L., et al. (2016). A novel CCM2 gene mutation associated with familial cerebral cavernous malformation. Front. Aging Neurosci. 8:220. doi: 10.3389/fnagi.2016. 00220

Labauge, P., Denier, C., Bergametti, F., and Tournier-Lasserve, E. (2007). Genetics of cavernous angiomas. Lancet Neurol. 6, 237-244. doi: 10.1016/s14744422(07)70053-4
Merello, E., Pavanello, M., Consales, A., Mascelli, S., Raso, A., Accogli, A., et al. (2016). Genetic screening of pediatric cavernous malformations. J. Mol. Neurosci. 60, 232-238.

Mondejar, R., Solano, F., Rubio, R., Delgado, M., Perez-Sempere, A., GonzalezMeneses, A., et al. (2014). Mutation prevalence of cerebral cavernous malformation genes in Spanish patients. PLoS One 9:e86286. doi: 10.1371/ journal.pone.0086286

Riant, F., Cecillon, M., Saugier-Veber, P., and Tournier-Lasserve, E. (2013). CCM molecular screening in a diagnosis context: novel unclassified variants leading to abnormal splicing and importance of large deletions. Neurogenetics 14, 133-141. doi: 10.1007/s10048-013-0362-0

Richards, S., Aziz, N., Bale, S., Bick, D., Das, S., Gastier-Foster, J., et al. (2015). Standards and guidelines for the interpretation of sequence variants: a joint consensus recommendation of the American college of medical genetics and genomics and the association for molecular pathology. Genet Med. 17, 405-424. doi: 10.1038/gim.2015.30

Rigamonti, D., Drayer, B. P., Johnson, P. C., Hadley, M. N., Zabramski, J., and Spetzler, R. F. (1987). The MRI appearance of cavernous malformations (angiomas). J. Neurosurg. 67, 518-524. doi: 10.3171/jns.1987.67.4.0518

Rigamonti, D., Hadley, M. N., Drayer, B. P., Johnson, P. C., Hoenig-Rigamonti, K., Knight, J. T., et al. (1988). Cerebral cavernous malformations. Incidence and familial occurrence. N. Engl. J. Med. 319, 343-347.

Scimone, C., Bramanti, P., Alafaci, C., Granata, F., Piva, F., Rinaldi, C., et al. (2017). Update on novel CCM gene mutations in patients with cerebral cavernous malformations. J. Mol. Neurosci. 61, 189-198. doi: 10.1007/s12031-016-0863-z

Scimone, C., Donato, L., Katsarou, Z., Bostantjopoulou, S., D'Angelo, R., and Sidoti, A. (2018). Two novel KRIT1 and CCM2 mutations in patients affected by cerebral cavernous malformations: new information on CCM2 penetrance. Front. Neurol. 9:953. doi: 10.3389/fneur.2018.00953

Shenkar, R., Shi, C., Rebeiz, T., Stockton, R. A., McDonald, D. A., Mikati, A. G., et al. (2015). Exceptional aggressiveness of cerebral cavernous malformation disease associated with PDCD10 mutations. Genet Med. 17, 188-196. doi: 10.1038/gim.2014.97

Spiegler, S., Najm, J., Liu, J., Gkalympoudis, S., Schroder, W., Borck, G., et al. (2014). High mutation detection rates in cerebral cavernous malformation upon stringent inclusion criteria: one-third of probands are minors. Mol. Genet. Genomic Med. 2, 176-185. doi: 10.1002/mgg3.60

Zafar, A., Quadri, S. A., Farooqui, M., Ikram, A., Robinson, M., Hart, B. L., et al. (2019). Familial cerebral cavernous malformations. Stroke 50, 1294-1301.

Zawistowski, J. S., Stalheim, L., Uhlik, M. T., Abell, A. N., Ancrile, B. B., Johnson, G. L., et al. (2005). CCM1 and CCM2 protein interactions in cell signaling: implications for cerebral cavernous malformations pathogenesis. Hum. Mol. Genet. 14, 2521-2531. doi: 10.1093/hmg/ddi256

Conflict of Interest: LH is employed by Running Gene Inc., Beijing, China.

The remaining authors declare that the research was conducted in the absence of any commercial or financial relationships that could be construed as a potential conflict of interest.

Copyright (c) 2021 Han, Ma, Qiao, Han, Wu and Li. This is an open-access article distributed under the terms of the Creative Commons Attribution License (CC BY). The use, distribution or reproduction in other forums is permitted, provided the original author(s) and the copyright owner(s) are credited and that the original publication in this journal is cited, in accordance with accepted academic practice. No use, distribution or reproduction is permitted which does not comply with these terms. 